\title{
LARGE DEFORMATION OF STRUCTURES BY SEQUENTIAL LIMIT ANALYSIS
}

\author{
WEI H. YANG \\ Department of Mechanical F.ngineering and Applied Mechanics, The University of Michigan, \\ Ann Arbor, MI 48109, U.S.A.
}

(Received 21 March 1991 ; in revised form 22 September 1992)

\begin{abstract}
Although most structures are designed to function under small deformation, their large deformation behavior can be used to estimate reliability and safety for survivorship from an accident or a natural disaster. Structures such as buildings, bridges, ships, vehicles and machinery are designed with a safety factor to protect certain assets from the unexpected and unknown elements. The large deformation analysis provides the rational basis for a safety factor. In this paper, the method of sequential limit analysis is used to compute large deformation solutions of truss and frame problems. Differing from the incremental method of plasticity, the limit analysis method is numerically stable, more effecient and requires simpler input data. A duality theorem serves as the foundation of an algorithm for computing the complete static and kinematic solutions simultaneously and for establishing their accuracy in each step of a deformation sequence. The phenomena encountered in large deformation such as loading-unloading under monotone deformation, bifurcation (more generally, loss of uniqueness) of solutions, and internal contact of structural members are revealed by the sequential limit analysis presented in this paper.
\end{abstract}

\section{INTRODUCTION}

Using limit analysis, the instantaneous velocity field of a truss or a frame structure undergoing plastic deformation is obtained for the current configuration. The velocity field is then integrated in a small time step to produce the displacement field which in turn updates the configuration of the deforming structure. In the case of a hardening material, local yield criteria are also updated. A subsequent limit problem is solved for this updated structure. This updating process is repeated to form a sequence leading to the solution of a large deformation problem. One major advantage of sequential limit analysis is global stability of computation.

Based on recent advances in limit analysis using duality theorems (Yang, 1987a, 1991) and computational optimization techniques (Luenberger, 1984), static and kinematic solutions for many complex problems can be obtained with a high degree of accuracy and certainty of convergence. A kinematic solution of a limit analysis problem can be interpreted either as a steady-state velocity field of a continuum in a Eulerian coordinate or instantaneous nodal velocities of the structure in a Lagrangian coordinate. Using the latter interpretation, the nodal velocity vector can be integrated in small time (or pseudo time) steps in a nonlinear cumulative sequence to produce a large deformation solution. Such solutions are sought in problems arising from structural reliability and failure analysis.

This nonlinear sequence may also be realized by an incremental analysis developed in connection with a finite element method [e.g. Lee et al. (1977)]. Such an approach, although widely used, has not been entirely satisfactory for reasons of high computing cost, uncertain accumulation of errors and numerical instability. An incremental method depends heavily on a one-to-one relation between stress and strain increments which may not exist in reality or may be ill-conditioned (a small change in input produces large change in output), causing numerical instability.

Limit analysis is a very efficient concept. It by-passes the tedium of keeping track of the details of incremental elastic-plastic constitutive equations and internal loading and unloading conditions. It uses an inequality form of constitutive relations. The rigorous convex analysis (Rockaffeller, 1970) and computational optimization techniques help to put limit analysis on sound mathematical foundation. The result is the recent surge of interest to reclaim limit analysis as a viable and general method for plasticity. Like the classical approach (Hodge, 1959), modern limit analysis uses a pair of related formulations to bound 
the exact solutions from above and below, but it is more mature in theory and methodology. It establishes a duality relation [e.g. Yang (1991)] that equates the least upper bound to the greatest lower bound. It uses computational optimization techniques to approach the corresponding maximum and minimum solutions simultaneously. A sensitivity analysis is used to detect bifurcation and, more generally, loss of uniqueness. Parametric limit analysis leads to optimal design of structures (Yang, 1978). In the case of large deformations, a sequential limit analysis is performed.

When the model of perfect plasticity is assumed, the sequential limit analysis may use just the upper bound formulation. Since static quantities do not enter the upper bound formulation explicitly, the sequence involves only geometric updating. accurate geometric updating can be achieved with relatively large step sizes compared with those used in incremental analysis in which complicated stress updating is also needed. This feature greatly improves computational efficiency, as tested in quite a few problems, by at least an order of magnitude from that of incremental analysis.

The hardening effect can also be included by using a yield criterion (an inequality relation) that varies with plastic deformation history. Actually, hardening is a stabilizing factor for incremental computation. For sufficiently large hardening, the matrix equation for incremental analysis remains well behaved thus stable incremental solutions can be computed easily. It is the lack of hardening (perfect plasticity) or presence of softening which causes difficulty in incremental computation. This is not the case for limit analysis in which perfect plasticity is assumed in all classical work on the subject. Limit analysis has been generalized to include a model called asymptotically perfect (Yang, 1982). Many ductile materials behave with rather insignificant hardening especially in large deformations. Even with hardening members, structures may still exhibit global softening in the form of increasing deformation under decreasing load due to nonlinear interaction between load and deformation. The sequential limit analysis remains globally stable under all material and geometrical nonlinearities. In cases where hardening is significant, its treatment in sequential limit analysis is still simpler than that in incremental analysis.

Of course, certain information, such as elastic strains, residue stresses and spring-back after load removal, may only be obtained by an elasto-plastic incremental analysis. But limit analysis can provide the most important information sought in structural mechanics at a fraction of the cost of incremental analysis. Residual stresses may still be obtained by an elastic unloading superimposed on a large deformation solution after it is obtained by sequential limit analysis.

We shall first present the primal (lower bound) formulation for the problems concerning limit analysis of structures with a specific constitutive inequality. Using the Hölder inequality, the constitutive inequality and the weak equilibrium statement, we derive the dual (upper bound) formulation. A duality theorem which equates the greatest lower bound to the least upper bound is then stated. This duality theorem is the basis of an algorithm (Yang, 1987b) for our numerical solutions.

Three examples are chosen to demonstrate the sequential limit analysis method and to reveal certain large deformation phenomena. Solutions of a three-bar truss, a bridge truss and a two-bay frame problems will bring out the thesis advocated in this paper without excessive computation to cloud the key issues. The computer program we have developed can handle up to 400 variables with ease on a microcomputer.

\section{VARIATIONAL FORMULATION OF LIMIT ANALYSIS}

Mechanics problems are governed by three fundamental principles: equilibrium of forces and moments, kinematics of deformation and constitution of materials. They are modeled by equations or inequalities so that a mechanics problem may be formulated and solved by mathematical techniques. When solutions of these equations and inequalities are interpreted as sets in an appropriate space, a methodology for solving a mechanics problem becomes a search for the intersection of the three fundamental sets. If the intersection set is empty, there is no solution to the problem posed. If it is a single point, the solution is unique, otherwise multiple solutions are admissible. The methodology of choosing the best 
among all admissible solutions is called optimization. Since most problems of this type must be solved numerically, computational methods of optimization have been developed and used as tools for obtaining limit analysis solutions (Huh and Yang, 1991).

We shall formulate and solve limit analysis problems of structures as a computational optimization problem. For the truss and frame structures made of one-dimensional members, the equilibrium condition in the current position of the structure can be expressed naturally by linear algebraic equations which balance the internal forces and moments in the members to the loads applied at the nodes, the junctions of the members. The equilibrium condition for the entire structure can be represented by a matrix equation :

$$
\mathbf{A t}=\lambda \mathbf{f},
$$

where $\mathrm{A} \in R^{m \times n}$ is an $m$ by $n$ matrix; $\mathbf{t} \in R^{n}$ is the unknown vector whose components comprise internal forces and moments in the members (e.g. a truss member carries only an axial force while a frame member may carry axial, shear forces and bending, twisting moments); $\lambda \mathbf{f} \in R^{m}$ is the vector of applied loads where $f$, a constant vector, may be normalized (e.g. $\|f\|_{2}=1$ ) and scaled by a scalar factor $\lambda \geqslant 0$. Components of $f$ are distributed on the nodes. The row dimension $m$ of $\mathbf{A}$ is usually smaller than the column dimension $n$. Otherwise, the system is either statically determinate or contains redundant equations. A static determinate problem is a simple one. The redundancy can be removed by an algebraic elimination process. A vector $t$ satisfying ( $1 a)$ is called statically admissible. All such vectors form the statically admissible set $S \subset R^{n}$.

Use of a load factor known as proportional loading was often regarded as being too restrictive to represent general loading conditions. This is not the case in our approach since the normalized vector $f$ can be changed and computation repeated to cover all desired load variations. Multiple scale factors and load vectors can also be used to parameterize complex load sequences. These details are considered at the programming stage.

We choose a simple constitutive model such that the asymptotic behavior of each component $t_{i}, i=1,2, \ldots n$ of the vector $\mathrm{t}$ is bounded above and below, $-l_{i} \leqslant t_{i} \leqslant u_{i}$, where $l_{i}$ and $u_{i}$ are material constants (e.g. buckling and tensile strengths for a truss member). If we centralize and normalize each $t_{i}$ with respect to its bounds, it is possible to bound every $t_{i}$ between -1 and 1 without loss of generality. This is achieved by a linear transformation on $\mathbf{t}$ which will also introduce in (la) a constant vector $\mathbf{g}$. Without changing notation for the transformed matrix $\mathbf{A}$, we rewrite (1a) as

$$
\text { At }=\lambda \mathbf{f}+\mathbf{g}
$$

where $\mathbf{g}$ is a constant vector which can be regarded as a dead load; while $\lambda \mathbf{f}$ is the live load which is allowed to increase or decrease by changing $\lambda$. If eqn (1a) already has a dead load term, then the constant vector from transforming $t$ can be added to it. We should note here that a dead load must be small enough for the structure to continue to carry more live load before it begins to collapse, otherwise, the limit analysis problem has no solution. This condition will show up in the existence proof not included in this paper.

Globally, the asymptotic behavior of the entire structure can be written as

$$
\|\mathbf{t}\|_{\infty}=\max _{i}\left\{\left|t_{i}\right|\right\} \leqslant 1 .
$$

Any vector $t$ satisfying (2) is called constitutively admissible and all such vectors form the constitutively admissible set $C \subset R^{n}$. Although we have not yet considered the deformation aspects of the structure, an exact static solution for a given applied load is contained in the intersection

$$
L=S \cap C .
$$

Since the equilibrium equations in (1b) are linear, the set $S$ is a convex hyperpolyhedron. 
The set $C$ is convex and bounded as dictated by the constitutive model (2). The intersection set $L$ is therefore convex and bounded. We further assume that the structure can at least carry the dead load without failure. There exists a small $\mathbf{t} \in L$ which is in equilibrium with g. This corresponds to the case $\lambda=0$. For small values of $\lambda$, there exist other values of $t$ which do not violate (2) and are elements of $L$. As $\lambda$ keeps increasing, the solution vector $\mathbf{t}$ will eventually reach an extreme point $\mathbf{t}^{*}$ of $L$. The corresponding $\lambda^{*}$ is called the limit load factor. Other points in $L$ always correspond to a load factor $\lambda \leqslant \lambda^{*}$. The set $L$ is appropriately called the lower bound solution set. A limit analysis problem seeks this extreme value $\lambda^{*}$ and the corresponding $\mathbf{t}^{*}$. It can be stated as a constrained optimization problem,

$$
\begin{aligned}
& \operatorname{maximize} \lambda \\
& \text { subject to } \mathbf{A t}=\lambda \mathbf{f}+\mathbf{g} \\
& \qquad\|\mathbf{t}\|_{\infty} \leqslant 1,
\end{aligned}
$$

which is a linear program although not in the so-called "standard form" (Luenberger, 1984). Since this general form of linear program appears frequently in limit analysis, we have constructed an efficient algorithm for large and sparse systems of this type. The solution to problem (4) known as the greatest lower bound consists of the limit load factor $\lambda^{*}=\max \lambda(t)=\lambda\left(t^{*}\right)$ where $t^{*} \in L$ is the maximizer sought. $\lambda^{*}$ is always unique because the constraint set $L$ is convex and bounded. But the solution vector $t^{*}$ may or may not be unique because the set $L$ contains flat boundaries.

The problem (4) has a dual problem in the form of another linear program whose minimum also equals $\lambda^{*}$. To derive the dual of (4), we use the weak equilibrium condition,

$$
\mathbf{v}^{\mathrm{t}}(\mathbf{A t}-\lambda \mathbf{f}-\mathbf{g})=\mathbf{0} \text { for all } \mathbf{v} \in K \subset R^{m},
$$

where the superscript $t$ transposes a vector or a matrix; $\mathbf{v}$ is the kinematic variable representing a possible velocity vector (rate of nodal translations and rotations for a general structure) in the admissible set $K$ in which all vectors satisfy prescribed kinematic boundary conditions. If (5) is satisfied for all vectors $v \in K$, then it is equivalent to (lb). The concept of weak equilibrium is also known as the virtual power principle in mechanics literature (Drucker, 1967). From (5), we obtain

$$
\lambda=\frac{\mathbf{v}^{\mathrm{t}}(\mathbf{A t}-\mathbf{g})}{\mathbf{v}^{\mathbf{t}} \mathbf{f}} .
$$

Since $\mathbf{v}$ appears homogeneously in both numerator and denominator of the expression above, it can be normalized such that $\mathbf{v}^{\mathbf{t}} \mathbf{f}=1$ with a unit of power. We may rewrite $\lambda$ in (6) and provide it with a sharp upper bound by the use of the Hölder inequality (Goffman and Pedrick, 1965) such that,

$$
\lambda=\mathbf{v}^{\mathbf{t}} \mathbf{A t}-\mathbf{g}^{\mathrm{t}} \mathbf{v}=\left(\mathbf{A}^{\mathrm{t}} \mathbf{v}\right)^{\mathrm{t}} \mathbf{t}-\mathbf{g}^{\mathrm{t}} \mathbf{v} \leqslant\left\|\mathbf{A}^{\mathrm{t}} \mathbf{v}\right\|_{1}\|\mathbf{t}\|_{\infty}-\mathbf{g}^{\mathrm{t}} \mathbf{v} \leqslant\left\|\mathbf{A}^{\mathrm{t}} \mathbf{v}\right\|_{1}-\mathbf{g}^{\mathrm{t}} \mathbf{v}=\lambda,
$$

where the upper bound $\lambda$ is a function of $\mathbf{v}$ and the $l_{1}$-norm of a vector $\mathbf{x}=\left(x_{1}, x_{2}, \ldots, x_{n}\right)$ is defined as $\|\mathbf{x}\|_{1}=\left|x_{1}\right|+\left|x_{2}\right|+\cdots+\left|x_{n}\right|$. The vector $\mathbf{A}^{\prime} \mathbf{v} \in R^{n}$ has the meaning of plastic deformation rate (e.g. rate of length changes in truss members or rotations of yield hinges in beams). Maximization of the inner product $\left(\mathbf{A}^{t} \mathbf{v}\right)^{t} t$ has been called the principle of maximum dissipation. Minimization of $\left\|\mathbf{A}^{\mathrm{t}} \mathbf{v}\right\|_{1}$ is known as the principle of minimum plastic deformation rate. These physical principles are subjected to preference of interpretations. An important mathematical result is that the maximum of one is equal to the minimum of the other. The proof of this duality relation is given in the paper on linear programming with bounded variables (Yang, 1992). Another "principle of consistency" in engineering literature simply states the fact that the components $\left(\mathbf{A}^{t} \mathbf{v}^{*}\right)_{i}$ and $t_{i}^{*}(i=1,2, \ldots, n)$ must 
have the same sign if $\left(\mathbf{A}^{t} \mathbf{v}^{*}\right)_{i} \neq 0$. In the language of linear programming, these conditions are called complementarity (Cottle et al., 1992). Here they arise naturally in the limit analysis of plasticity. If the signs of the corresponding components above are different, they produce a negative term in $\lambda$; therefore, the value of $\lambda$ can still be improved in the maximizing sense by a solution that satisfies the complementarity condition.

The dual problem seeks the least upper bound in the form:

$$
\begin{aligned}
& \operatorname{minimize} \bar{\lambda} \\
& \text { subject to } \bar{\lambda}=\left\|\mathbf{A}^{\mathbf{t}} \mathbf{v}\right\|_{1}-\mathbf{g}^{\mathrm{t}} \mathbf{t} \\
& \mathbf{v}^{\mathbf{t}} \mathbf{f}=1,
\end{aligned}
$$

which is also a linear program in the form of $l_{1}$-norm minimization. Our algorithm solves (4) and (8) simultaneously to provide complete static and kinematic solutions. The duality theorem provides

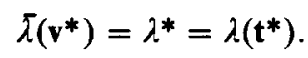

The choices of $\mathbf{v}^{*}$ and $\mathbf{t}^{*}$ that satisfy (9) may violate certain notions of continuity or smoothness of solutions. For instance, kinking (yielding hinge) in a beam is not an admissible form of velocity in linear elasticity. A correct theory of plasticity must redefine the set of kinematical admissibility. The set $K$ must be enlarged (Cesari and Yang, 1991) to include certain nonsmoooth functions. The correct $K \subseteq R^{m}$ here is the one which produces the duality relation (9) and gives the correct interpretation of $\mathbf{v}^{*} \in K$. The duality relation (9) must be proved for each class of problems [e.g. plates (Yang, 1987a), plane stress (Huh and Yang, 1991), torsion (Yang, 1991) etc.] in their respective $K$. Again $\mathbf{v}^{*}$ and $\mathbf{t}^{*}$ may or may not be unique.

The computed value of $\mathbf{v}^{*}$ is based on the current configuration of the structure. We may integrate this current velocity to obtain the displacement vector which will cause the structure to change shape. Since the plasticity theory we use is rate independent, a quasistatic displacement can be written as $\mu \mathbf{v}^{*}$ where $\mu$ is another scale factor called step size (a pseudo time increment). A choice of $\mu$ should give the displacement the unit of length and be small enough to make the nonlinear geometric change approximately affine. We require

$$
\mu^{(k)}\left\|\mathbf{v}^{*}\right\|_{\infty} \ll \mathrm{L}, \quad k=0,1,2, \ldots
$$

where $k$ is the step index and $L$ is a characteristic length of the structure. The condition (10) validates small deformation theory within each step. The displacement vector $\mu \mathrm{v}^{*}$ is then used to update the configuration of the structure. When this procedure is repeated, a cumulative sequence leads to a large deformation solution. This is similar to the Euler's method (Milne, 1957) for a system of ordinary differential equations (ODE). Mimicking a multistep ODE method, the current velocity vector may also be combined with those obtained in several previous steps to form a more accurate displacement vector for the current step. We choose the simpler method for the examples presented in the following three sections to put emphasis on phenomena of large deformation rather than on integration methods. In each step, the static solution in terms of internal forces and moments and the kinematic solution in terms of nodal velocity are obtained simultaneously from the primal-dual algorithm.

The method presented in this paper applies to three-dimensional structures with general hardening properties. But the examples presented in the following three sections are twodimensional with asymptotically perfect plasticity so that important issues in large plastic deformations are brought to light without distracting and nonessential complications. 


\section{A PLANAR THREE-BAR TRUSS}

A three-bar truss with a $45^{\circ}$ inital configuration and a characteristic length $L$ as shown in Fig. 1 is subjected to an $x$-direction load (tension or compression) applied at the joint node. The step size is chosen to be $0.01 \mathrm{~L}$. To combine the tension and compression results in a single graph, we begin in compression then continue into tensile modes by moving the node to the right, through the plane $(x=0)$ of fixed nodes. The motion is animated by superposing a sequence of the deformed truss in dash lines as shown in the bottom of Fig. 1 with the moving node highlighted as black dots. At the beginning of the compression, a bifurcation causes deformation to undergo an unsymmetric mode as the node moves below or above the $x$-axis. It is worth noting here that no instruction is given to force symmetry in the computer program. The minimization procedure chooses a correct unsymmetric mode since the symmetric mode corresponds to a higher load. Symmetry arguments used in many linear analysis may not apply in limit analysis.

As the node moves to the right in the compression phase, the load factor decreases. When the node passes through the plane $x=0$, the load factor drops to zero then increases as the truss enters into the tensile phase. The load factor is plotted against the $x$-displacement $u$ of the moving node as shown by the $\lambda^{*}$-curve above the animation.

One may intuitively expect the tensile deformation of the truss to proceed smoothly and to reach a symmetric mode. But slight chatter is observed in the load-displacement curve in Fig. 1 as the displacement $u$ reaches beyond $2 \mathrm{~L}$. This is not a numerical error. Since the three-bar truss will deform plastically when the center bar and one of the side bars reach their yield limits, producing an unsymmetric deformation. Unlike the bifurcation in the compressive phase, this unsymmetric deformation is self correcting. As the moving node passes through the plane of symmetry $(y=0)$, equilibrium will cause a switch of the yielding members and reverse the $y$-direction velocity. This chattering motion can also be detected in the trace of the moving node in the animation. The load-displacement curve asymptotically approaches the value 3 from below as expected when the three bars reach near parallel positions.

This seemingly simple problem has revealed some complex characters of large deformation which are oblivious to researchers in linear theories of structures. In particular,

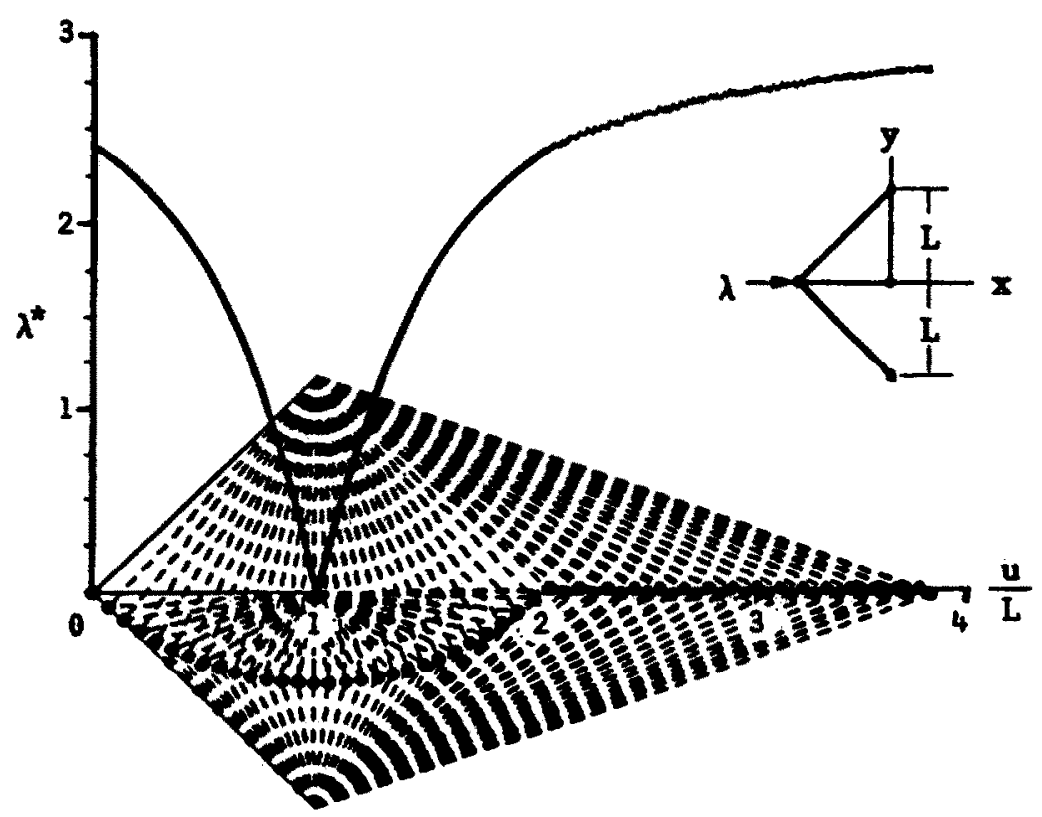

Fig. 1. Deformed configurations and load-displacement curve of the three-bar truss. 
the assumed symmetry, all too often used in computational projects to reduced domain representation, cannot be taken matter-of-factly. Decreasing load under monotone deformation may appear as a softening behavior but it is not caused by the softening material property.

\section{A PLANAR BRIDGE TRUSS}

For the simple three-bar truss, the bifurcation leads to two entirely different deformation in compression and produces nonunique but nearby solutions (chatters) in tension. When more complex structures are considered, small perturbations in strength, geometry of different members and in load distributions may lead to a large number of different solutions. Even in small deformation theory of limit analysis, it is well known that a single collapse load may correspond to many collapse modes. This lack of uniqueness can be easily explained from the convex optimization view point. The optimality of a convex function over a convex set is always unique but the optimizer as a boundary point of the set may not be. For large deformation analysis, initial nonuniqueness may lead to many entirely different subsequent solutions. Initial uniqueness may still branch later under small perturbations.

To further explore the above stated characteristics of large deformation, we consider a larger structure in the form of a bridge truss shown in Fig. 2 with 15 identical members of length $L$ joined at 9 nodes. Three equal downward forces are applied at nodes, 2, 3 and 4.

In Fig. 3, node 3 is shown as black dots in a sequence of the deforming bridge truss. The truss first deforms in a symmetric mode. Then node 3 begins swaying to the right as deformation proceeds. There is no data bias to cause this unsymmetric deformation. It is a case of bifurcation. Later in the sequence, the swaying stops and node 3 begins to move toward the center position. When the vertical displacement of node 3 reaches beyond the value $2 \mathbf{L}$, horizontal chatter of node 3 begins while vertical motion continues downward as shown by the black dots in Fig. 3. This chattering behavior is also evident in the loaddeformation curve in Fig. 4 when $v_{3}>2 \mathrm{~L}$. These phenomena are essentially the same as that presented in the three-bar truss problem except that now there may be more deformation modes.

We choose two slightly different input data to produce two quite different deformation sequences. In case $I$, the load on node 2 is made slightly lower (by $0.1 \%$ ). This causes the deformation to initially sway to the left. The subsequent deformation is the mirror image to that shown in Fig. 3. This minute load bias causes the truss to choose one branch of the bifurcation. Case II involves a higher buckling strength of the bar linking nodes 7 and 8 , therefore the input data remains symmetric. The stronger bar 7-8 causes initial yielding to take place elsewhere (bar 2-6). These two altered deformation sequences are shown side by side in Fig. 5 in the form of a few snapshots at equal intervals in the sequences. The bars

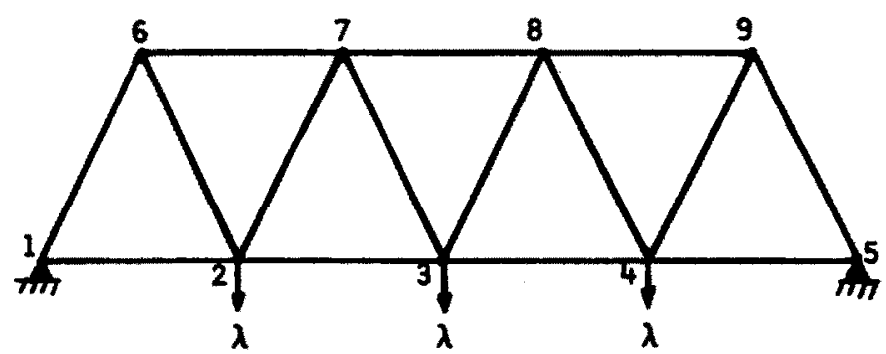

Fig. 2. A bridge truss and applied loads. 


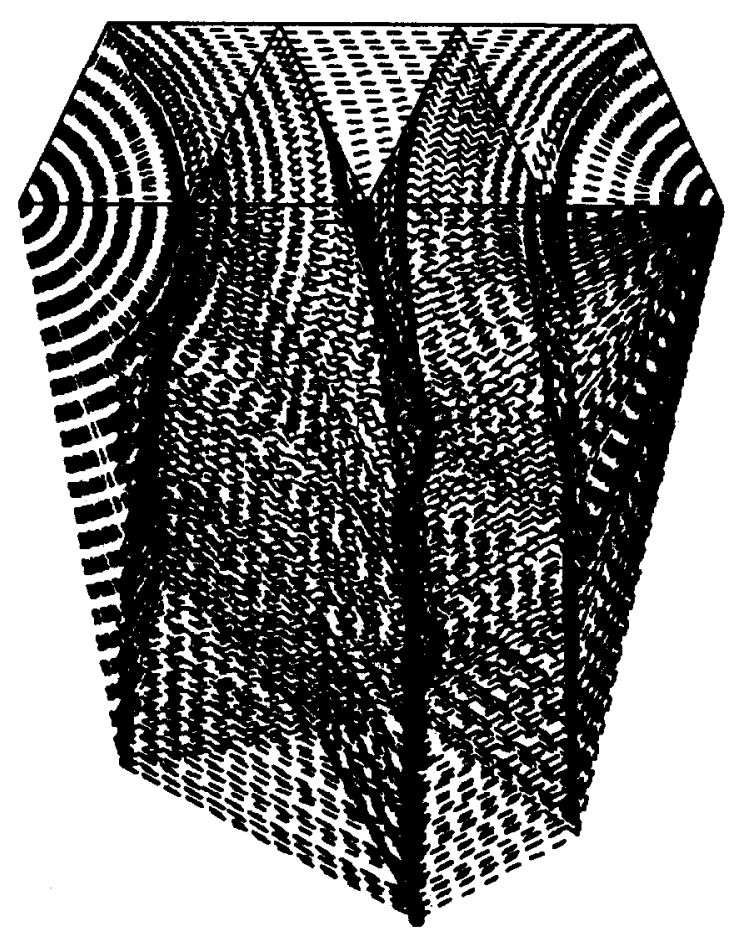

Fig. 3. A deformation sequence of the bridge truss.

shown by dash lines are undergoing tensile yielding ; the bar with the label " $c$ " is undergoing compressive yielding or buckling; the bars shown in solid lines are moving as rigid bodies.

The load-deformation curve of case $I$ is identical to that shown in Fig. 4 while that of case II differs only slightly. This reconfirms one assertion in limit analysis that the unique collapse load may correspond to many collapse modes. From the mathematical viewpoint, it is clear that many extreme points of a convex set in the lower bound formulation (4) may lie on a flat boundary. They and their convex combinations are all maximizers of the

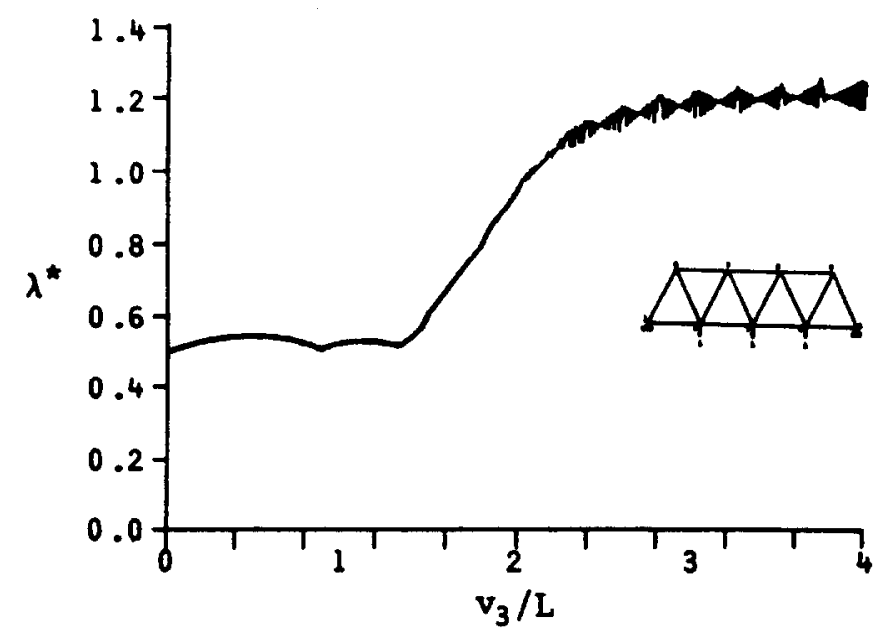

Fig. 4. A load-deformation curve of the bridge truss. 

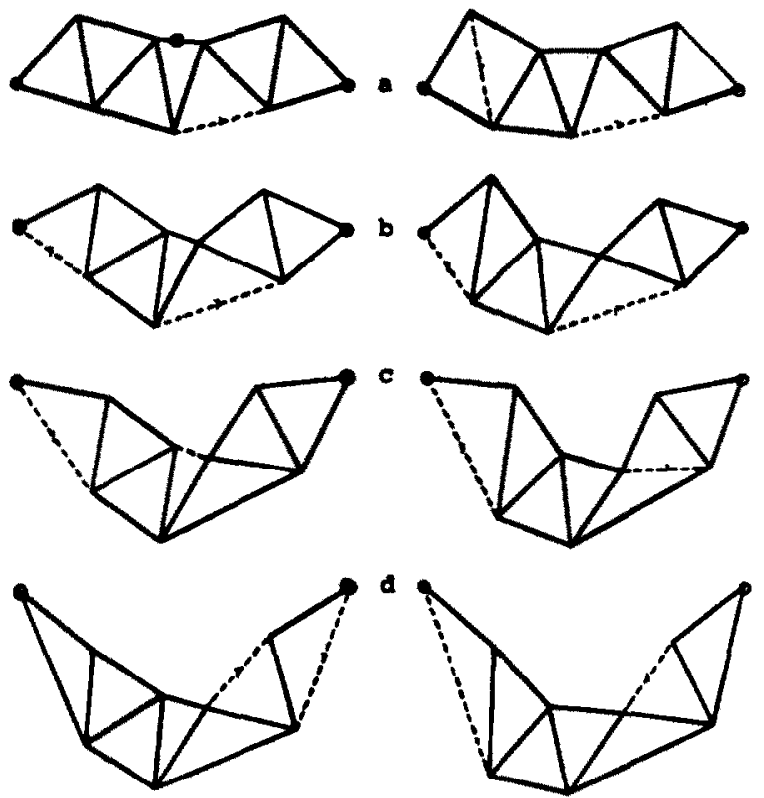

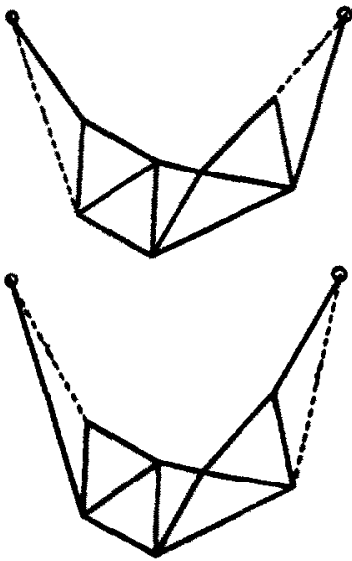

I

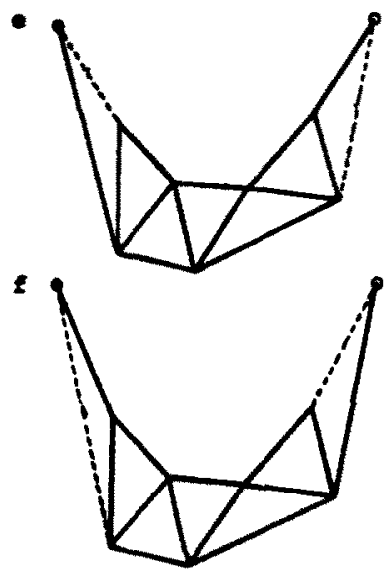

II

Fig. 5. Two deformation sequences of the bridge truss.

objective function. Similarly, the $l_{1}$-norm function in the upper bound formulation (8) may have a flat bottom that contains multiple minimizers.

\section{A PLANAR TWO-BAY FRAME}

A frame is a welded structure using beams as its elemental members. The loads on it are assumed to be or are made equivalent to a set of point forces so that its algebraic equilibrium equations are exact. A member of the frame is a straight beam element whose location in the frame is determined by the coordinates of its end points. We assume no applied loads in the interior of an element and the ends of the elements connect at the nodes where loads can be applied. If a load is applied at an interior point of a beam element, a node is added there and the element is divided into two. A preprocessor generates the equilibrium equations computationally using basic data of the elements and loads as input whose format is made similar to that of NASTRAN finite element programs. The static variables in a plane frame element consist of the bending moments and shear forces at the two ends and the axial force in the element. For three-dimensional frames, twisting moments are also present. 
For a plane frame, the equilibrium conditions consist of three equations for each member and for each node. Since shear failure is uncommon in frames, we can eliminate all shear forces in the system and reduce the number of equations in (1b) in the process. The variable $t$ now consists of two nodal moments and an axial force for each member. A constitutive model provides upper and lower bounds for each component of $t$. The resulting lower bound formulation, after normalizing the bounds of $t$, is in the form of (4) and its dual is in the form of (8). The dual variable $\mathrm{v}$ now consists of nodal velocities and rotation rates.

The two-bay frame shown in Fig. 6(a) has 10 elements and 11 nodes. Nodes 1, 6 and 11 are anchored into the ground (built-in). Three vertical and two horizontal loads are applied at nodes $3,7,9,2$ and 10 with respective magnitudes $\frac{3}{2} \lambda, \lambda, \lambda, \lambda$ and $\frac{1}{2} \lambda(\lambda \geqslant 0)$. The asymptotic bending strength of all members is $M_{0}$ except for the right-bay roof beams whose bending strength is $2 M_{0}$. The axial and shear strength of the members are assumed to be large enough to not require a bound (or an infinite bound), an assumption made here for simplicity and for an exact comparison to an example in Harrison (1979). We use the length $L$ of member $1-2$ as the characteristic length of the frame. The primal and dual problems are solved simultaneously giving the static solutions in terms of the member forces and moments, and the kinematic solutions in terms of nodal velocities and rate of rotations. The first-step solution agrees with that of Harrison's solution.

When we commence a sequence, the nodal velocities are multiplied by a scale factor (step size) to obtain the nodal displacements. The scale factor is chosen so that the member 1-2 rotates $1^{\circ}$ in each step. The displacements of all nodes so obtained have magnitudes in the order of $0.01 \mathrm{~L}$ and are used to update the nodal positions and thus the current geometry of the deforming frame. Seven deformed configurations of the frame at intervals of $6^{\circ}$ rotations of member 1-2 are shown in solid lines in Figs $6(b-h)$. The dashed lines provide a comparison to the previous configuration. A step size of $6^{\circ}$ rotation (a six-fold increase) produced slightly less accurate but quite satisfactory results. This confirms the conjecture that sequential limit analysis is much less demanding for small step size than the incremental analysis for computing accurate, large plastic deformations.

In each step, plastic deformation is concentrated at certain nodes known as the yield hinges (Hodge, 1959). The limit load factor decreases with increasing deformation in the beginning as shown by the curve in Fig. 7. This apparent softening behavior of the frame is the result of nonlinear load-geometry interaction. This softening effect can cause trouble
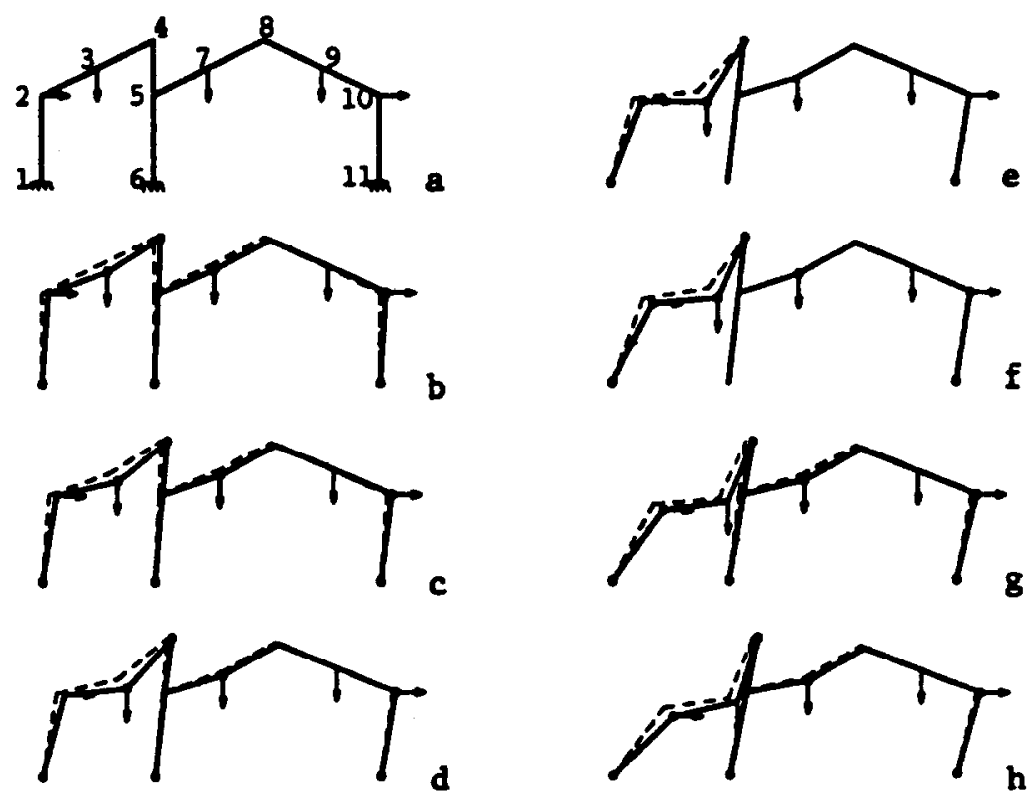

Fig. 6. A two-bay frame and its deformations. 


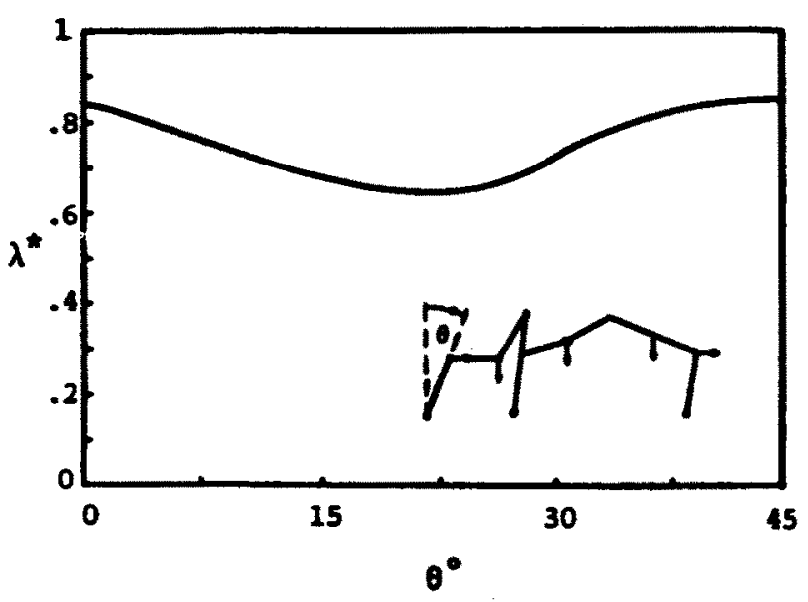

Fig. 7. Load-deformation curve of a two-bay frame.

for an unsophisticated incremental method but does not hinder sequential limit analysis. Deformation proceeds initially under the same mode in which seven yield hinges at nodes $1,3,4,6,7,10$ and 11 are formed as shown in Figs $6(\mathrm{~b}-\mathrm{d})$. Between the steps $\mathrm{d}$ and $\mathrm{e}$, the 7-hinge mode changed to a 5-hinge $(1,2,3,4,11)$ mode. Local unloading at nodes 6 , 7 and 10 has obviously occurred. In the meantime, the global load factor begins to increase, showing a hardening effect. Between the steps $e$ and $f$, the left-bay of the frame continues to deform plastically while the right-bay stays stationary. But at step $f$, hinges reform at nodes 7 and 10. From there on, deformation proceeds in a different 7-hinge mode. We have shown again the phenomenon of loading, unloading and reloading (certain plastic hinges appear, disappear then reappear in the frame) under a monotonic deformation sequence. Soon after step $h$, node 3 is about to collide with member 5-6. We stop the computation, not wanting to add the complication of internal contact to the scope of this paper although it can certainly be included in the sequential limit analysis.

This sequence of large deformation solutions has captured phenomena of apparent global softening, hardening, local unloading, reloading and possible internal contact all under a simple, monotone deformation of the two-bay frame whose members are assumed perfectly plastic. These are all realistic phenomena of large deformation. For more complex structures, cycles of loading-unloading become more frequent and may occur in many locations. This can be a serious problem for an incremental method since stress updating in a chattering sequence may require very small increments and frequent domain-wide searches for loading or unloading elements. One may still encounter an ill-conditioned tangent stiffness matrix.

\section{OBSERVATIONS AND CLAIMS}

Limit analysis enjoyed a burst of intense development in the 1950s but stopped short of making a sweeping presence, as it should have, in modern structural analysis. There are two reasons for the subsequent decline of interest in limit analysis. Some students were led to believe, although wrongly, by the terminology "rigid-perfectly-plastic" used in earlier publications on the subject, that the model is simplistic and unrealistic. On the other hand, researchers were confronted by the mathematical and computational difficulties unresolved at the time even for the "simple" model. Consequently, limit analysis seemed to have the attribute of a narrow and specialized topic in structural mechanics. Yet, its validity extends to the asymptotic behavior of all metal structures.

"Rigidity" is an unnecessary assumption and the perfectly-plastic model realistically represents the asymptotic behavior of many ductile materials. In fact, such behavior has been regarded so desirable that research laboratories worldwide have prototyped many superplastic alloys for various structural applications and are now gearing up for their 
commercialization. Our recent work extends sequential limit analysis to hardening and softening materials.

On the theoretical front, recent advances in convex analysis and optimization methods as well as the vastly improved computing facilities have removed many obstacles which previously impeded the development of limit analysis. We have used those improvements and extended them to sequential limit analysis for general structure and continuum problems. The examples in this paper are only intended to demonstrate how limit analysis is applied to large plastic deformations. Much larger and more complex problems can now be solved by sequential limit analysis using microcomputers.

Like other asymptotic methods in mechanics, limit analysis can obtain important information with much reduced input requirements. The relaxed demand here applies to constitutive modeling. Instead of a detailed one-to-one incremental elastic-plastic stressstrain relation, the asymptotic behavior is modeled by an inequality. There is no loss of generality in this representation since the elastic range is implied in the constitutive inequality although not explicitly modeled. Furthermore, the limit solutions are independent of the intermediate elastic-plastic behavior. The hardening (and softening) behavior of a member can be easily modeled by a change of strength at the end of each step, according to how much plastic deformation the member has accumulated. This stepwise change of strength can fit any experimental data. Microscopically, dislocation movements in crystalline materials resemble a stepwise slip-stick motion. The tangent modulus used in incremental analysis is only a model of the observed hardening phenomenon rather than the true microscopic behavior.

In theory, an incremental solution of ductile structure approaches a limit asymptotically and should converge to the limit solution. In practice, the matrix equation for incremental analysis becomes ill-conditioned while approaching the limit, causing inaccuracy. This difficulty is intrinsic to any linearized method when the original nonlinear problem admits multiple solutions. If a matrix equation admits nonunique solutions, the matrix must be singular. The limit analysis will produce computationally "exact" solutions even though they are not unique. The sequential limit analysis presented in this paper, clearly demonstrated by examples, is an accurate and efficient tool for the large deformation analysis of structures.

One of the troublesome but challenging aspects of large deformation plasticity is the loss of uniqueness. To explore the range of multiple solutions, a sensitivity study by perturbation must be performed. Each limit analysis step is a nonlinear problem. A sequence leading to large deformation may consist of dozens of steps (or hundreds of increments in the case of incremental analysis). A perturbation study requires multiple sequences. A typical structural analysis problem usually involves a vector variable of several hundred unknowns. One can easily imagine the immense magnitude of the computational task. Without an efficient methodology to carry out this task, many theoretical claims on large deformation analysis seem rather hollow. With the state of the art of the finite element method, most incremental solutions presented in literature typically consist of a single sequence of increments, a symmetrically reduced domain, and artifice of economizing and stabilizing schemes of computation. Few of these solutions can be rigorously verified. If one tries experimental verification, the task is even greater than the computational project itself since every test sequence is destructive to the specimen. Nonunique or near nonunique solutions suggest difficulty in repeatability of the experiments.

The sequential limit analysis is mathematically rigorous. It is numerically accurate, efficient and stable. It offers new means for through investigations and new hope for deeper understanding of large plastic deformations.

\section{REFERENCES}

Cesari, L. and Yang, W. H. (1991). Serrin integrals and second order problems of plasticity. New directions in differential equations and dynamic systems. Roy. Soc. Edinburgh 117A, 193-207.

Cottle, R. W., Pang, J. S. and Stone, R. E. (1992), Linear Complimentarity Problem. Academic Press, New York. Drucker, D. C. (1967). Introduction to Mechanics of Deformable Solids. McGraw-Hill, Maidenhead.

Goffman, C. and Pedrick, G. (1965). First Course in Functional Analysis. Prentice-Hall, Englewood Cliffs, NY. 
Harrison, H. B. (1979). Structural Analysis and Design, Part I. Pergamon Press, Oxford.

Hodge, P. G., Jr (1959). Plastic Analysis of Structures. McGraw-Hill, Maidenhead.

Huh, H. and Yang, W. H. (1991). A general algorithm for limit solutions of plane stress problems. Int. J. Solids Siructures 28(6), 727-738.

Lee, E. H., Mallett, R. L. and Yang, W. H. (1977). Stress and deformation analysis of the metal extrusion process. Comp. Meth. Appl. Mech. Engng 10, $339-353$.

Leunberger, D. G. (1984). Linear and Nonlinear Programming. Addison-Wesley, Reading, MA.

Milne, W. E. (1957). Numerical Solution of Differential Equations. Wiley, New York.

Rockaffeller, R. T. (1970). Convex Analysis. Princeton University Press.

Yang, W. H. (1978). On a class of optimization problems for framed structures. Comp. Meth. Appl. Mech. Engng $15,85-97$

Yang, W. H. (1982). A variational principle and an algorithm for limit analysis of beams and plates. Comp. Meth. Appl. Mech. Engng 33, 575-582.

Yang, W. H. (1987a). A duality theorem for plastic plates. Acta Mech. 69, 207-212.

Yang, W. H. (1987b). A general computational method for limit analysis. ASME-PVP 129, 21-31.

Yang, W. H. (1991). A duality theorem for plastic torsion. Int. J. Solids Structures 27(15), 1981-1989.

Yang, W. H. (1992). Linear program of bounded variables (to appear). 